Bundesgesundheitsbl - Gesundheitsforsch Gesundheitsschutz 2004 · 47:611-621

DOI 10.1007/s00103-004-0857-9

๑) Springer-Verlag 2004

R. Kurth · Robert Koch-Institut, Berlin

\title{
Das Auftreten alter und neuer Seuchen als Konsequenz menschlichen Handelns
}

der auf die transfusionsrelevanten Infektionen wie Syphilis, Hepatitis und HIV eine zunehmende Infektionssicherheit bei Bluttransfusionen erreicht werden.

Ein radikales Umdenken bei Ärzten, Wissenschaftlern und im öffentlichen Gesundheitsdienst setzte ein, als AIDS 1981/82 als neuartige Infektionskrankheit diagnostiziert werden konnte und wenig später feststand, dass es sich hierbei um eine virale Erkrankung handelt, die primär sexuell sowie über Blut und Blutprodukte übertragen wird. Verstärkt wurde diese Bewusstseinsänderung auch durch das Auftreten neuer, bisher unbekannter Infektionen wie der Bovinen Spongiformen Enzephalopathie (BSE) der Rinder, vorwiegend in England Mitte der 8oerJahre. Bei dieser Krankheit bemerkte man wenig später, dass es sich um eine Infektionskrankheit handeln musste, die nach heutigem Kenntnisstand offenbar von einem kontagiösen Eiweiß, einem pathogenen Prion, verursacht wird.

\section{(2) Die derzeitige Prävalenz der Infektionskrankheiten ist weltweit gesehen unakzeptabel hoch}

Dieses Umdenken in der Infektionsmedizin führte zu der Erkenntnis, dass in den letzten Jahrzehnten eine ganze Reihe von Infektionen als klinisch neue Erkrankungen definiert werden mussten (• Übersicht 1). Daraus resultiert die Erkenntnis, dass wir auch in Zukunft mit neuartigen Infektionskrankheiten rechnen müssen und dass Epidemien mit bisher unerkannten Erregern oder eine Aus- breitung bekannter Erreger zu erwarten sind. Instrumentarien und internationale Netzwerke, vor allem der Weltgesundheitsorganisation (WHO), müssen ausgebaut werden, um neue Epidemien oder gar Pandemien möglichst früh zu erkennen und zu bekämpfen. In diesem Zusammenhang ist anzumerken, dass die derzeitige Prävalenz der Infektionskrankheiten weltweit gesehen ohnehin unakzeptabel hoch ist (• Tabelle 1 ). Während parasitäre und Infektionskrankheiten in den Industrieländern für weniger als $1 \%$ aller Todesursachen verantwortlich sind, beträgt dieser Anteil in den Entwicklungsländern immer noch über $40 \%$.

Die ausschlaggebenden Faktoren für die Renaissance altbekannter und das Auftreten neuer Seuchen und deren Konsequenzen werden im Folgenden aufgeführt. Es ist wieder einmal das Handeln des Menschen, das Risiken erhöht und in diesem Fall die Ausbreitung von Infektionserregern erleichtert. Da von allen Mikroorganismen Viren als evolutionär am erfolgreichsten eingestuft werden müssen und, vereinfachend zusammengefasst, mit ihren hohen Mutationsraten Selektionsvorteile besonders effektiv und schnell realisieren können, steht die zukünftige Entwicklung von viralen Infektionskrankheiten im Mittelpunkt dieses Beitrags.

\section{Schwierigkeiten in der Infektionsbekämpfung}

Impfstoffe gelten als die effektivsten und preiswertesten Arzneimittel, die man 
Tabelle 1

Globale Mortalitätsstatistik 2001: die wichtigsten Todesursachen

(WHO-Health Report 2002, http://www.who.int)

\begin{tabular}{|lcc}
\hline & Fälle (Millionen) & Prozentsatz \\
\hline Herz-/Kreislauf-Erkrankungen & 16,7 & 29,2 \\
\hline Infektionen (einschließlich parasitäre) & 15,0 & 26,2 \\
\hline Krebs & 7,1 & 12,5 \\
\hline Unfälle, Selbstmord, Gewalt, Kriege & 5,2 & 9,1 \\
\hline Kindersterblichkeit (Perinatal conditions) & 2,5 & 4,3 \\
\hline Lungenerkrankungen & 3,7 & 6,5 \\
\hline Erkrankungen der Verdauungsorgane (z. B.Leberzirrhose) & 2,0 & 3,4 \\
\hline Neuropsychiatrische Erkrankungen & 1,1 & 1,9 \\
\hline Diabetes mellitus & 1,0 & 1,7 \\
\hline Erkrankungen der Genitale und Nieren & 0,8 & 1,5 \\
\hline Schwangerschaftsbezogene Todesfälle (Maternal conditions) & 0,5 & 0,9 \\
\hline Nährstoffmangel (z. B.Jod, Vitamin A) & 0,5 & 0,8 \\
\hline
\end{tabular}

Tabelle 2

Der Einfluss erfolgreicher Impfstoffe (USA). (Aus: Folkers u. Fauci, Nat Med 4, 1998; bzW. MMWR 2001/Vol.50/No.53)

\section{Erkrankung}

Diphtherie

Masern

Mumps

Keuchhusten

Kinderlähmung

Röteln

\section{Gipfel der Inzidenz}

206.939 (1921)

894.134 (1941)

$152.209(1968)$

265.269 (1934)

$21.269(1952)$

$57.686(1969)$
Fälle 2001

2

116

266

7.580

0

23 kennt (• Tabelle 2). Jedoch stehen für viele Infektionskrankheiten wie AIDS, Hepatitis $\mathrm{C}$ oder Rotavirusinfektionen, die in Entwicklungsländern bei Säuglingen eine hohe Mortalität verursachen, noch keine Impfmöglichkeiten zur Verfügung. Auch gegen viele bakterielle Infektionen fehlen effektive Vakzine. Noch gravierender in quantitativer Hinsicht ist, dass es bisher keinen einzigen Impfstoff gegen eine parasitäre Infektion gibt.

Angesichts von 2 Millionen Malariatodesfällen pro Jahr ist die Entwicklung eines Impfstoffs gegen Malaria daher ebenso erstrebenswert wie ein Impfstoff gegen AIDS. Mehr als ärgerlich ist weiterhin, dass aus finanziellen Gründen Impfstoffe häufig dort nicht zur Verfügung stehen, wo sie am dringendsten benötigt werden, vor allem in Ländern der Dritten Welt. Man muss sich auch darüber im Klaren sein, dass viele Infektions- krankheiten ausgerottet werden könnten, wenn die gefährdete Population weltweit geimpft würde. Dies ist 1977 bereits mit der Ausrottung der Pockenviren gelungen und wird wahrscheinlich innerhalb dieses Jahrzehnts auch beim Poliovirus, dem Erreger der Kinderlähmung erreicht. Weiterhin könnten Erkrankungen wie Hepatitis-B-Infektionen, wie Masern, Mumps und Röteln durch weltweite konsequente Impfung ausgerottet werden, da es für diese Viren kein Tierreservoir gibt.

Ein weiteres Problem in der Infektionsbekämpfung ist der unsachgemäße Einsatz von Antibiotika sowohl in der Humanmedizin als auch als Leistungsförderer in der Tiermast, der die Resistenzentwicklung bei Bakterien fördert. Es ist ein Phänomen der letzten 20 Jahre, dass zunehmend Bakterienstämme in Krankenhäusern diagnostiziert wurden, die multi- resistent gegen zuvor wirksame Antibiotika geworden sind.

Entwicklungen der modernen Intensivmedizin wie der Einsatz von Zytostatika oder die Unterdrückung der immunologischen Abstoßung von Transplantaten führten zu einer steigenden Zahl von iatrogen immunsupprimierten Patienten, die durch opportunistische und andere Infektionen in einem besonderen Maß gefährdet sind. Auch hier sehen wir also eine Situation, in der (medizinischer) Fortschritt eine Erregerausbreitung begünstigt.

\section{Infektionskrankheiten im Wandel}

Im angelsächsischen Sprachraum spricht man seit einigen Jahren von den „Emerging Infectious Diseases“ oder gar von „Emerging Epidemics“. Damit bezeichnet man die Tatsache, dass Infektionskrankheiten weltweit gesehen quantitativ im Anstieg begriffen sind und dass zunehmend neue Infektionskrankheiten oder neue humanpathogene Erreger diagnostiziert werden. Von Menschen verursachte Risiken sowie eine mangelnde Technologiefolgenabschätzung erleichtern den Infektionserregern das Leben. $\mathrm{Zu}$ den Risiken gehören

- technologische Fortschritte,

- Veränderungen der Umwelt,

- Veränderungen im Lebensstil und stark erhöhte individuelle Mobilität/ Reisetätigkeit,

- bioterroristische Anschläge.

\section{Risiken durch technologischen und medizinischen Fortschritt}

Beispielhaft kann hier die Landwirtschaft erwähnt werden, die sich einerseits weltweit schnell entwickeln muss, um eine wachsende Bevölkerung ernähren zu können, die andererseits aber auch unter einem ständigen Kosten- und Wettbewerbsdruck steht. Die dafür notwendigen technologischen Entwicklungen umfassen unter anderem eine ausgeprägte Mechanisierung sowie den Einsatz von Herbiziden und Insektiziden, die unvermeidlich $\mathrm{zu}$ Veränderungen in Flora und Fauna in den Anbaugebieten führen. Virusstämme, die auf Zwischenwirte (Vektoren) an- 
gewiesen sind, erfahren durch diese Eingriffe häufig eine zunehmende Verbreitung. Hierzu nur ein einziges von zahllosen Beispielen: Die starke Ausweitung des Kakaoanbaus in Mittel- und Südamerika seit Mitte der 6oer-Jahre führte zu einer zunehmenden Verbreitung eines viralen hämorrhagischen Fiebers (Oropouche-Fieber), dessen Erreger ein durch Stechmücken übertragenes Bunyavirus ist. Die infizierten Stechmücken fanden für ihre Vermehrung in mit Regenwasser gefüllten Kakaoschalen ideale Brutbedingungen. Die „Therapie“ war in diesem Fall einfach: Kakaoschalen durften nicht auf den Feldern liegen bleiben.

Eine Intensivierung der Massentierhaltung zur Versorgung mit animalen Proteinen war erst möglich, nachdem die gefährlichsten Tierseuchenerreger entweder durch Impfung oder durch Tierbestandssanierungen unter Kontrolle gebracht werden konnten (z. B. Tuberkulose, Brucellose). Die Intensivierung der Tierhaltung, insbesondere durch gleichzeitige Aufzucht verschiedener Arten, bringt die Gefahr mit sich, dass Viren durch Spezieswechsel eine veränderte pathogene Bedeutung für Mensch und Tier erlangen können.

Influenzaviren sind hierfür ein typisches Beispiel. Wirklich neue, durch Impfung mit aktuellen Impfstoffen praktisch nicht abzuwehrende und damit gefährliche Influenzavirusvarianten entstehen immer dann, wenn sich entweder im Tier (z. B. Schwein) oder im Menschen nach Doppelinfektionen mit humaner und aviärer Influenza durch Neukombinationen (Reassortment) neuartige Influenzavarianten bilden, gegen die dann keine Populationsimmunität vorliegt. Schweine sind das klassische „Mischgefäß“ humaner und aviärer Influenzaviren. Daher ist die z. B. in Südchina übliche gleichzeitige Haltung von Geflügel und Schweinen ein ständiges Bedrohungspotenzial. Bei dem in seinem Ausmaß historisch einmaligen Ausbruch der Geflügelpest (Influenza A/ $\mathrm{H}_{5} \mathrm{~N}_{1}$ ) Anfang des Jahres 2004 in fast einem Dutzend asiatischer Staaten wurde der intensive Kontakt mit Tieren auch direkt gefährlich. Eine Reihe von Menschen starb nach engem Kontakt zu infiziertem Geflügel. Bereits 1997 hatte $\mathrm{H}_{5} \mathrm{~N} 1$ erstmals direkt auf den Menschen über-

Bundesgesundheitsbl - Gesundheitsforsch - Gesundheitsschutz 2004 - 47:611-621 DOI 10.1007/s00103-004-0857-9

(C) Springer-Verlag 2004

R. Kurth

\section{Das Auftreten alter und neuer Seuchen als Konsequenz menschlichen Handelns}

\section{Zusammenfassung}

Im Zeitalter der Antibiotika und Impfstoffe wurde vor dem Auftreten von AIDS den bekannten Infektionskrankheiten nicht selten eine abnehmende klinische Bedeutung beigemessen. Darüber hinaus wurde zuweilen die Ansicht vertreten, dass bisher unbekannte Infektionskrankheiten kaum noch zu erwarten seien. Bei genauer Analyse der Lage der Infektiologie ergibt sich jedoch ein sehr anderes Bild. In den letzten Jahrzehnten sind zahlreiche neuartige Infektionskrankheiten klinisch erkannt worden. Neue humanpathogene Viren, Bakterien und Parasiten sind entdeckt worden, altbekannte

haben ihre klassischen Verbreitungsgebiete verlassen. Vor allem virale Erreger können sich auch ständig genetisch verändern und dadurch neue Relevanz für die Klinik erhalten. Der Beitrag zeigt, dass es in erster Linie der Mensch ist, der durch sein Handeln Auftreten und Verbreitung von Infektionskrankheiten in der Vergangenheit beeinflusst hat und in der Zukunft bestimmen wird.

\section{Schlüsselwörter}

Neue Infektionen · Evolution von Erregern . Risikofaktor Mensch

\section{Human factors in emerging infectious diseases}

\section{Abstract}

In the era of antibiotics and vaccines and prior to the appearance of AIDS, well-known infectious diseases received decreasing clinical attention. Occasionally, the opinion was also expressed that new types of infectious diseases could no longer be expected. However, a more detailed analysis of the state of infectious diseases yields quite a different picture. A variety of new infectious diseases has clinically been defined over the last few decades. New viruses, bacteria, and parasites with pathogenic potential for humans have been detected and well-known microorganisms have spread beyond their original geographic areas. Infectious agents, in particular viruses, permanently alter their genomes and may thus gain new clinical relevance. This article demonstrates that primarily the behavior of man influenced the nature and distribution of infectious diseases in the past and will affect the spread of infectious diseases in the future.

\section{Keywords}

Emerging infectious diseases .

Evolution of infectious agents · Risk factor man 
gegriffen, in Hongkong, sodass damit auch der Mensch selbst „Mischgefäß“ für aviäre und humane Influenzaviren sein kann.

Durch Massentierhaltung kann sich aviäre Influenza in Geflügelfarmen rasch verbreiten. Ein großer Ausbruch vom Subtyp A/H7N7 fand im Februar 2003 in den Niederlanden statt. Es wird vermutet, dass freilaufendes Geflügel sich am Kot von durchziehenden Wildenten, die mit $\mathrm{A} / \mathrm{H}_{7} \mathrm{~N}_{7}$ infiziert waren, angesteckt hatte. Mehr als 260 Menschen mit engem Kontakt zu erkranktem Geflügel bekamen eine Bindehautentzündung, bei etwa einem Viertel von ihnen wurde das aus dem erkranktem Geflügel stammende Influenzavirus nachgewiesen; ein Tierarzt starb an der Erkrankung. Bei Schweinen in Massentierhaltung, konnte aufgrund serologischer Untersuchungen eine Infektion mit dem Virus nachgewiesen werden, ohne dass es bei den Tieren zu Erkrankungen kam.

Auch das Auftreten von BSE (Rinderwahnsinn) und der neuen Variante der Creutzfeldt-Jakob-Krankheit (vCJD) sind Ergebnisse von Änderungen in der Tierfütterung. Es steht außer Frage, dass aus der Schlachtung und Tierkörperbeseitigung gewonnene proteinreiche Tiermehle sowie möglicherweise auch Tierfette, die bei der Rinderfütterung eingesetzt wurden und unerkannt den BSE-Erreger enthielten, das wichtigste Vehikel für die Ausbreitung der Krankheit waren. Das infektiöse Agens war dabei nur ungenügend inaktiviert worden. Die bisher rund 140 Fälle von vCJD, ganz vorwiegend im Vereinigten Königreich aufgetreten, können plausibel dadurch erklärt werden, dass diese Menschen sich durch den Genuss von Nahrungsmitteln infiziert hatten, die aus BSE-infizierten Tieren hergestellt worden waren.

Auch der ständig steigende internationale Handel führt zu einem Anstieg importierter Infektionskrankheiten. Futtermittel- und Rinderexporte aus dem Vereinigten Königreich haben in anderen europäischen Ländern zum Auftreten von BSE geführt. Der internationale Handel von Affen, vor allem zu Forschungszwecken, und in früheren Jahrzehnten noch nicht so reguliert wie heute, hat wiederholt zu Infektionen des wissenschaftli- chen Personals in den Empfängerinstituten geführt. Bekanntestes Beispiel ist die Infektion von Wissenschaftlern am PaulEhrlich-Institut in Frankfurt und bei den Behringwerken in Marburg durch so genannte Filoviren aus afrikanischen Grünen Meerkatzen, die 1968 importiert worden waren. Aus den Affen und infizierten Menschen wurde das kausale Agens, später als Marburg-Virus bezeichnet, isoliert. Die Infektionen verliefen schwer und zum Teil tödlich.

Auch therapeutische und prophylaktische Fortschritte in der Humanmedizin resultieren in eindrucksvollen Beispielen für neue Transmissionswege von Erregern. Dies trifft insbesondere für die Transfusionsmedizin zu. So wurden bereits in den Jahren 1911 und 1915 die ersten Berichte über Malaria- und Syphiliserkrankungen im Zusammenhang mit Transfusionen veröffentlicht, und eine 1943 publizierte Arbeit beschrieb bereits das gehäufte Auftreten von Gelbsucht 34 Monate nach einer Transfusion. Nach Entdeckung des Hepatitis-B-Virus und der Entwicklung spezifischer Nachweisverfahren konnte auch eine Untersuchung der Blutspender auf das Hepatitis-B-Oberflächenantigen erfolgen, und viele transfusionsassoziierte Infektionen konnten dadurch verhindert werden. Es sollte jedoch noch bis 1989 dauern, bis auch das Hepatitis-C-Virus als häufigster Auslöser der „Non-A-non-B-Hepatitis“ entdeckt wurde. Durch die Einführung der Untersuchung von Blutspenden auf Anti-HCV 1990 sank die Zahl transfusionsassoziierter HCV-Infektionen erheblich. Seit 1999, mit Einführung des HCVGenomnachweises, wurde dem Paul-Ehrlich-Institut kein solcher Fall mehr gemeldet. Noch vor der Entdeckung des für AIDS ursächlichen Erregers „HIV“, Anfang der 8oer-Jahre des vergangenen Jahrhunderts erkannte man aufgrund epidemiologischer Zusammenhänge, dass diese Immunschwäche auch transfusionsassoziiert auftreten konnte.

Strikte Spenderauswahlkriterien, um infektiöse Personen von der Blutspende auszuschließen, und die Einführung der Testung von Blutspendern auf transfusionsrelevante Infektionen sowie Maßnahmen zur Virusinaktivierung von Plasmaderivaten haben wesentlich dazu beige- tragen, dass heute das Risiko, eine Syphilis, Hepatitis oder HIV-Infektion durch Gabe von Blut oder Blutprodukten zu erwerben, in den Industriestaaten verschwindend klein geworden ist.

Neue Infektionsrisiken könnten aber auch durch Xenotransplantation, die Übertragung von tierischen Zellen, Geweben oder Organen auf den Menschen, entstehen. Die favorisierten Spendertiere sind Schweine - wegen ihres Stoffwechsels, der dem des Menschen ähnelt, der vergleichsweise großen mikrobiologischen Sicherheit, der Organgröße und aus Kostengründen. Die weit fortgeschrittene Charakterisierung von Viren des Schweins, z. B. Herpesviren, und die Entwicklung empfindlicher Methoden zum Nachweis dieser Erreger hat wichtige Grundlagen gesetzt für die Züchtung von Tieren, die solche Erreger nicht besitzen.

Während die meisten Erreger durch Auswahl und geeignete Haltung der Schweine beseitigt werden können, ist das für so genannte PERVs nicht möglich. Diese „porcinen endogenen Retroviren" sind im Erbgut aller Schweine verankert. PERVs können menschliche Zellen infizieren. In einer Versuchsreihe wurde kürzlich im Robert Koch-Institut gezeigt, dass Affen, denen große Mengen an porcinen endogenen Retroviren verabreicht wurden und deren Immunsystem ähnlich wie bei einer Transplantation unterdrückt wurde, nicht mit dem Virus infiziert worden sind. Auch bei Affen, die Organe vom Schwein erhalten hatten, und bei Patienten, die erste klinisch-experimentelle Xenotransplantationen erhielten, z. B. Inselzellen des Schweins bei Diabeteskranken, konnte keine Übertragung von PERVs nachgewiesen werden. Doch können die möglichen Risiken der Xenotransplantation nach wie vor noch nicht vollständig abgeschätzt werden.

\section{Risiken durch Veränderungen in der Umwelt}

Mittlerweile ist der Mensch in fast jeden Winkel der Erde vorgedrungen, d. h., er kam mit vielen Spezies erstmals in Kontakt. Dadurch wurde es möglich, dass zoonotische Erreger von seltenen Tierar- 
ten auf den Menschen übergehen konnten (Transspezies-Transmission).

Die Affenpocken können hier zur Illustration herangezogen werden. Affenpocken traten in Zentralafrika praktisch nur dort auf, wo umfangreiche Waldrodungen vorgenommen worden waren. Der Übertragungsweg ist nicht eindeutig geklärt. Wahrscheinlich ist der Name irreführend, da offenbar einheimische Nager die natürlichen, nicht krank werdenden Wirte dieser Virusfamilie sind und Affen genauso wie Menschen durch diese Viren als heterologe Wirte infiziert werden können. Bisher gingen die Fachleute davon aus, dass der Mensch zwar individuell infiziert werden kann, das Virus aber nicht auf andere Menschen überträgt. Besorgnis erregend ist eine Entwicklung aus den letzten Jahren, dass offenbar Affenpockenviren durchaus von Mensch zu Mensch übertragen werden können, wenngleich (noch?) mit niedriger Effizienz.

Die Situation in Zentralafrika ist deshalb potenziell gefährlich, weil weltweit vor nun fast 30 Jahren die Pockenvirusimpfung berechtigterweise eingestellt wurde. Die junge Bevölkerung wäre gegen ein Affenpockenvirus, das sich an den Menschen adaptiert hätte, nicht geschützt. Bei den mit Kuhpockenvirus geimpften älteren Menschen scheint nach infektionsepidemiologischen Untersuchungen noch ein gewisser Schutz zu bestehen. Das Gefahrenpotenzial durch Affenpocken wird von der Weltgesundheitsorganisation und anderen einschlägigen Instituten genau beobachtet. Die potenzielle Bedrohung durch Affenpocken, auch in den Industriestaaten, bestätigte ein Ausbruch in den USA im Frühjahr 2003, bei dem sich über 30 Personen mit dem Virus infiziert hatten. Ursächlich war die Haltung von Präriehunden als Haustiere, die sich in einer Tierhandlung bei aus Afrika importierten Nagern infiziert hatten.

\section{(7) Fernstraßenbau und Wasserwirt- schaft fördern die Ausbreitung von Erregern}

Fernstraßenbau kann zur Ausbreitung derjenigen Erreger führen, die in den neu erschlossenen und zuvor nur sehr we- nig bevölkerten Siedlungsgebieten nicht vorhanden waren. Der Bau der Transamazonica in Brasilien erlaubte die Ausbreitung der Malaria in das zentrale Amazonasbecken. Anfang der 8oer-Jahre erfuhr HIV seine schnellste Ausbreitung entlang der Fernstraße von Kampala nach Mombasa. Wasserwirtschaft, also unter anderem Staudammbau und landwirtschaftliche Bewässerungsprojekte, erlauben die Ausbreitung sowohl parasitärer Erkrankungen wie Malaria, Schistosomiasis und Bilharziose als auch von Viren, die von Mücken als Zwischenwirt abhängig sind. Stauseen in den Tropen sind wegen ihrer parasitären Verseuchung grundsätzlich für den Menschen nicht zum Baden geeignet.

\section{Mobilität und Lebensstil als Risikofaktoren}

Die überwiegend erst nach dem Zweiten Weltkrieg neu entstandenen Megastädte in den Entwicklungsländern mit ihrer sehr hohen Bevölkerungsdichte und ihren unzureichenden öffentlichen Hygieneeinrichtungen sind für die Entstehung von Epidemien prädestiniert. Diese Städte besitzen normalerweise keine flächendeckende einwandfreie Trinkwasserversorgung und Abwasseraufbereitung, sodass Infektionen mit Enterobakterien und Enteroviren endemisch und praktisch unvermeidbar sind. Hinzu kommt, dass offene Wasserbehälter ideale Brutstätten für Mückenlarven darstellen, die mit unterschiedlichen Viren infiziert sein können, die von Mücken auf Menschen übertragbar sind (z. B. Gelbfiebervirus, Dengue-Virus). Ratten und andere Nagetiere können ebenfalls zahlreiche unterschiedliche Bakterien- und Virusstämme übertragen (z. B. Lassa-Virus).

Promiskuität ist keine Erfindung der Neuzeit. Dennoch hat die in einigen industrialisierten und Drittweltländern gesellschaftlich tolerierte oder ignorierte $\mathrm{Li}$ beralisierung sexueller Verhaltensweisen zu einer Zunahme sexuell übertragbarer Infektionskrankheiten geführt. AIDS ist dafür nur ein Beispiel, auch die anderen klassischen venerischen Krankheiten sind keinesfalls rückläufig. Auch intravenöser Drogenmissbrauch kann natürlich $\mathrm{zu}$ einer effektiven Verbreitung sol- cher Erreger führen, die durch Blut übertragbar sind.

\section{(> Mikroorganismen „reisen“ am effizientesten in infizierten Tieren oder Menschen}

Truppenverschiebungen, Flüchtlinge, Emigranten und natürlich auch Urlauber können für den Transport von humanpathogenen Erregern sorgen. Die Medizingeschichte ist voll von Berichten darüber, wie das Aufeinandertreffen bisher getrennt lebender Populationen zur Erregerausbreitung führt. Klassische Beispiele sind die Einschleppung der Syphilis durch die Truppen von Columbus nach ihrer Rückkehr aus Zentralamerika und der Pocken durch Cortes und seine Truppen nach Südamerika. Die Einschleppung der Masern auf die Falklandinseln zu Beginn des letzten Jahrhunderts und auf die Faröerinseln Ende der 3oer-Jahre führte zu schweren Epidemien unter der einheimischen Bevölkerung, die zuvor noch nie mit Masernviren in Berührung gekommen war. In diesem Zusammenhang ist an die Gefährdung von Fernurlaubern, die in tropische Gebiete reisen, zu erinnern. Sie können Infektionskrankheiten wie Malaria, Gelbfieber, Hepatitiden oder auch HIV in ihre Heimat zurückbringen.

Die Überwindung weiter Strecken ist sowohl für aerogen übertragene Erreger als auch in kontaminiertem Wasser für fäkal-oral übertragene Erreger nur wenig effizient. Auch die Medizingeschichte zeigt, dass die Ausbreitung von Seuchen durch infizierte Menschen und Tiere am effizientesten war. Die Beulenpest übersprang leicht weite Entfernungen durch infizierte Ratten auf Schiffen und brach im Mittelalter immer wieder primär in Hafenstädten aus. Das Gelbfiebervirus kam offenbar mit dem Sklavenhandel nach Amerika. Das Dengue-Virus erreichte den amerikanischen Kontinent, weil gebrauchte Autoreifen aus Thailand importiert wurden, in denen sich Regenwasser angesammelt hatte, das Moskitolarven enthielt. Die Larven und die sich daraus entwickelnden Moskitos waren mit dem Dengue-Virus infiziert.

Auch 2 Epidemien aus jüngster Zeit machen deutlich, wie leicht Infektions- 
erreger „auf Reisen“ gehen können: Die seit 1999 herrschende Westnil-Virusepidemie in den USA ist vermutlich durch importierte Vögel oder Insekten ausgelöst worden. Das Westnil-Virus infiziert über Moskitos aber nicht nur Vögel, sondern auch eine Reihe anderer Tierarten sowie den Menschen. Bei Menschen verlaufen die meisten Infektionen inapparent, aber bei etwa jedem Fünften kommt es zu dem grippeähnlichen Westnilfieber. Ein kleiner Teil der Betroffenen erkrankt an einer Enzephalitis, die tödlich enden kann. In den USA starben seit Beginn der Epidemie mehrere hundert Menschen durch das Westnilvirus.

$\mathrm{Zu}$ Beginn des Jahres 2003 schließlich wurde ein neuartiges Coronavirus nicht zuletzt durch den intensiven weltweiten Flugverkehr zur neuen und unerwarteten Bedrohung für die Menschheit. Ausgehend von einem initial kleinen Infektionsherd in einer chinesischen Provinz „reiste“ der Verursacher des schweren akuten Atemwegssyndroms (SARS) in rund 30 Staaten und führte zu etwa 8.400 Infektionen und mehr als 900 Todesfällen.

\section{Bioterroristische Anschläge als Risikofaktor}

Pockenviren sollten sich offiziellen Angaben zufolge nur noch in den Hochsicherheitslaboratorien der USA (in Atlanta) und Russlands (in Koltsowo, Nowosibirsk) befinden. Es ist jedoch nicht auszuschließen, dass noch in anderen Laboratorien Pockenviren vorhanden sind. Die WHO hat nie zertifiziert, ob 1980 alle Laboratorien, die mit Pockenviren gearbeitet hatten, diese tatsächlich vernichteten. Im Umfeld der großen Umwälzungen in der ehemaligen Sowjetunion wurde der Umzug des Hochsicherheitslabors von Moskau nach Nowosibirsk ebenfalls mit Sorge betrachtet. Die Verantwortlichen haben zugegeben, dass es nie eine Inventur gegeben hat. Vor diesem Hintergrund sah sich das Robert Koch-Institut nach dem 11. September 2001 und den Milzbrandbriefen im Oktober desselben Jahres in den USA veranlasst, Vorsorge zu treffen und der Bundesregierung $\mathrm{zu}$ empfehlen, Pockenimpfstoff für die gesamte Bevölkerung zu bevorraten. Noch im Oktober 2001 richtete die Bundesregierung am Robert Koch-Institut eine „Zentrale Informationsstelle des Bundes für Biologische Sicherheit“ (IBBS) ein. Inzwischen ist diese Informationsstelle Teil eines Zentrums für Biologische Sicherheit geworden, in dem neben der Modellierung von Szenarien zur Abwehr bioterroristischer Anschläge insbesondere die Schnelldiagnostik relevanter Erreger und Toxine auf- bzw. ausgebaut wird (s. auch Bundesgesundheitsblatt November 2003 mit dem Themenschwerpunkt Biologische Sicherheit).

\section{Bedingungen für Ausbreitungs- erfolge von Infektionserregern}

Ein Erreger kann nur dann sein Verbreitungsgebiet erweitern, wenn es ihm gelingt, geographische Barrieren, die Populationen trennen, zu überwinden. Gelingt dies einem Erreger, so muss seine Kontagiosität so groß sein, dass er in einer neuen Population nach transienten Infektionen nicht immunologisch eliminiert werden kann. Dies ist immer dann der Fall, wenn dem Erreger bei seiner geographischen Ausbreitung die gleiche Wirtsspezies, z. B. der Mensch, zur Verfügung steht. Versucht jedoch ein Erreger eine Speziesbarriere zu überwinden, $d$.h. eine neue Tierart oder erstmals den Menschen zu infizieren, so sind diese Erreger initial nur schlecht an den neuen Wirt adaptiert, und nur in den seltensten Fällen kommt es zu einer dauerhaft erfolgreichen Transspezies-Transmission (• Übersicht 2).

Bei seinem Ausbreitungsbestreben ist es für einen Mikroorganismus keinesfalls von evolutionärem Vorteil, eine besonders hohe Pathogenität an den Tag zu legen. Es könnte die Gefahr bestehen, dass der Erreger seine neue Wirtspopulation ausrottet und damit selbst zum Aussterben verurteilt ist. Als zu Beginn der 5oer-Jahre die Kaninchenpocken (Myxomatose) absichtlich in Australien eingeführt wurden, um der dort herrschenden Kaninchenplage Herr zu werden, betrug die Letalität der Infektion für Kaninchen etwa 99\%. Diejenigen Kaninchen, die die Infektion überlebten, bevölkerten mit ihren Nachkommen langsam wieder den Kontinent, erreichten allerdings nur ein Viertel der ursprünglichen Populations- dichte. Für diese Population ist die Myxomatose nur noch zu etwa $20 \%$ letal. Mit anderen Worten: Es hat sich ein neues Gleichgewicht zwischen der Populationsdichte des Wirts und der Letalität des Erregers eingestellt.

\section{Schritte in der Erregermanifestation}

Aufgrund genetischer und epidemiologischer Überlegungen kann davon ausgegangen werden, dass wirklich neuartige Erreger in der Natur nur sehr selten entstehen. Natürlich verändern sich viele Mikroorganismen ständig (• Übersicht 3), als neu oder neuartig sollte man jedoch nur solche Erreger kennzeichnen, deren genomische Veränderungen zu einem leicht messbaren Selektionsvorteil (z. B. veränderter Tropismus, veränderte Pathogenität) geführt haben.

Insbesondere Viren besitzen ein sehr plastisches Genom, d.h. die Fähigkeit zur schnellen genomischen Veränderung. Punktmutationen ereignen sich permanent bei der Replikation von DNA und noch viel häufiger bei der Vermehrung von RNA, weil die RNA-abhängigen Polymerasen kein Korrektursystem gegen den irrtümlichen Einbau eines falschen Nukleotids in eine wachsende RNA- oder DNA-Kette besitzen (• Tabelle 3).

Viren mit ihrem normalerweise extrem kleinen Genom können sich durch genomische Deletionen, durch Rekombinationen und durch Gensegmentaustausche schnell verändern. So gut wie immer führen diese genomischen Veränderungen zu einer reduzierten Fitness oder gar zur Letalität des Mikroorganismus, sind für das Virus also nicht von Vorteil. Diesen scheinbaren Nachteil kompensieren Viren durch ihre hohe Replikationsrate, und gelegentlich kommt es eben $\mathrm{zu}$ jenen genomischen Veränderungen, die zu einer erhöhten Fitness führen. Letztere kann auch die Pathogenität erhöhen oder reduzieren (letzteres ist viel häufiger) oder zu einem veränderten und erweiterten Tropismus führen.

Punktmutationen können ebenfalls $\mathrm{zu}$ Veränderungen der Immunogenität von Viren führen. Die Influenzaviren sind hierfür das bekannteste und epidemiologisch wichtigste Beispiel. Solche Punktmutationen werden auch als Esca- 
pemutanten bezeichnet, man findet sie z. B. bei Hepatitis-B-Viren oder bei Retroviren. Durch Punktmutationen können neutralisierende Epitope verändert werden, d.h., die Mutanten können wenigstens vorübergehend der Neutralisation durch Antikörper entgehen, bis die humorale Immunität die Mutanten wieder erkannt hat. Sind die Mutanten hochkontagiös und treffen sie auf eine Population, die durch frühere Infektionen nicht gefeit ist, so können sie sich besonders schnell im Sinne einer Epidemie oder Pandemie ausbreiten. Für die Mikroorganismen ist die Rekombination neben der Mutation eine weitere Möglichkeit, veränderte Nachkommenschaft zu bilden. Bei Viren muss es zur Doppelinfektion einer einzigen Zelle kommen, damit die Genome der Elternviren rekombinieren können. Bakterien haben bekanntlich als zumeist extrazellulär wachsende Mikroorganismen andere Mechanismen des Genaustausches entwickelt, z. B. den Transfer von Erbmaterial durch Konjugation, d. h. durch Übertragung auf die bakterielle Nachbarzelle, oder über Phagen. Wie bei den Punktmutationen haben rekombinante Nachkommen nicht unbedingt einen Selektionsvorteil, es sei denn, sie können schneller replizieren als die Eltern, haben einen erweiterten Tropismus, oder sie haben, z. B. bei Bakterien, ein Antibiotikaresistenzgen aufgenommen.

Viren mit einem segmentierten Genom wie die Orthomyxoviren, zu denen auch die Influenzaviren gehören, können durch Segmentaustausch mit anderen Stämmen ihrer Virusfamilie ihre Immunogenität und ihren Tropismus drastisch und schnell ändern. Wie bereits oben erwähnt, kann es bei Doppelinfektionen in einer Wirtszelle zum Segmentaustausch kommen. Pandemien der Influenzaviren erfolgen meist nach einem Austausch von Genen von Virusstämmen, die natürlicherweise in verschiedenen Wirten heimisch sind.

Veränderungen im Genom, insbesondere bei den genomisch kleinen Viren mit ihrer hohen Replikationsrate, garantieren noch lange nicht, dass ein Erreger evolutionär erfolgreicher sein wird als seine viralen Vorfahren. Im 20. Jahrhundert haben wir jedoch immer wieder erleben können, dass ein bisher unbekanntes
Übersicht 1

Kleine Auswahl klinisch neu erkannter Infektionen (etwa seit 1960)

Bakterielle Infektionen

B-Streptokokken

Campylobacter

Helicobacter

Legionellen

Yersinien

Bartonella

Borrelien

\section{Virale Infektionen}

Viren der hämorrhagischen Fieber, z. B. Marburg-Virus, Ebola-Virus, Lassa-Virus, Hantaan-Virus

FSME-Virus (Frühsommer-Meningoenzephalitis)

Humane T-Zell-lymphotrope Viren (HTLV-I,-II)

Humane Immundefizienzviren (HIV-I,-2)

Humane Herpesviren (z. B. HHV-6 bis -8)

Infektionen durch Proteine (Prionen)

Kuru des Menschen

Neue Variante der Creutzfeldt-Jakob-Krankheit (vCJK)

Infektionen durch Pilze

Penicillium marneffei

Scedosporium prolificans

Cunninghamella bertholletiae

Übersicht 2

Kriterien für den evolutionären Erfolg von Infektionserregern

Verfügbarkeit empfänglicher Wirte (und wenn notwendig empfänglicher Zwischenwirte)

Dauer der Infektion (akut, chronisch, persistierend)

Leichtigkeit der Übertragbarkeit

Leichtigkeit der geographischen Ausbreitung durch mobile Wirte

Optimale Korrelation zwischen Übertragbarkeit und Virulenz

Übersicht 3

Molekulare Mechanismen der mikrobiellen Evolution

Punktmutationen (alle Erreger, besonders häufig bei RNA-Viren)

Rekombinationen (alle Erreger, häufig z. B. bei Retroviren; Alphaviren)

Genetisches Reassortment (Segmentaustausch bei Orthomyxoviren, z. B. Influenza; Bunyaviren)

Genetische Konjugation und Transduktion, inkl. Transfer von Antibiotikaresistenzund Virulenzgenen (Bakterien)

Übersicht 4

Schritte in der Virusmanifestation

1. Das Virus entwickelt sich in der Natur

2. Das Virus hat sich im Tier etabliert, ist aber bisher ohne Kontakt zum Menschen

3. Ausgeprägte Exposition und Infektion des Menschen führt zu einer neuen Erkrankung

4. Bereits bekannte Viren verursachen neuartige Erkrankungen

5. Bereits bekannte Viren ohne (zumindest erkennbares) pathogenes Potenzial

6. Bekannte Infektionskrankheiten mit unbekannter, vermutlich viraler Ätiologie 
Virus, das z. B. eine einzige oder nur wenige Tierspezies als natürlichen Wirt besitzt, in umfangreichen Kontakt mit Menschen kam und sich an den Menschen adaptieren konnte (• Übersicht 4 ). Eine solche Wirtsbereichserweiterung kann, muss aber nicht zur Erkrankung führen, da viele Infektionen mit Viren, Bakterien und auch Parasiten relativ apathogen verlaufen.

In den letzten Jahrzehnten konnten auch immer wieder Mikroorganismen (s. $\bullet$ Übersicht 1), die bereits bekannt waren, als Erreger von Krankheiten identifiziert werden. Ein umfangreich publiziertes Beispiel ist die chronische Infektion mit Helicobacter pylori, einer Bakterienspezies, die mittlerweile unbestritten für die chronische Gastritis verantwortlich ist, die langfristig sogar zur Entwicklung eines Magenkarzinoms führen kann.

Darüber hinaus gibt es eine große Anzahl von Mikroorganismen, die den Menschen zwar infizieren können, aber nicht humanpathogen zu sein scheinen. Umgekehrt gibt es jedoch auch Infektionskrankheiten, deren Ursache bisher nicht bekannt ist. Letztlich gibt es auch noch Erreger, die sich unserem molekularbiologischen Verständnis, d. h. unseren Vorstellungen über ihre Replikationsstrategien und über ihre Pathogenitätsmechanismen, weitgehend entziehen. Dazu gehören z. B. die Viroide („nacktes“ Erbmaterial, meistens RNA ohne jede Proteinhülle) oder die Prionen (infektiöse Eiweiße). Viroide verursachen häufig ökonomisch verheerende Pflanzenkrankheiten, sie können Plantagen vernichten. Pathogen gefaltete Prionen führen zu den gefürchteten zentralnervösen Degenerationen, den schwammartigen Gehirnerweichungen, wie z. B. zur Bovinen Spongiformen Enzephalopathie (BSE), zu Scrapie der Schafe oder zur neuen Variante der CreutzfeldtJakob-Krankheit des Menschen.

\section{Parameter für den evolutionären Erfolg von Viren}

Natürlich muss eine Virusfamilie auf eine ausreichende Anzahl von Angehörigen ihrer Wirtsspezies treffen, um ihr eigenes Überleben sicherzustellen (s. auch - Übersicht 2). Letztlich müssen die Viren mehr Individuen infizieren können, als infizierte Individuen sterben. Einige Viren erreichen dieses Ziel dadurch, dass sie nach Infektion lebenslang chronischpersistierend im Wirt verbleiben (gilt für Herpesviren, Retroviren). Bei Viren, die auf Zwischenwirte wie Mücken oder Nager angewiesen sind, ist zusätzlich notwendig, dass diese Zwischenwirte in ausreichender Anzahl zur Verfügung stehen.

\section{( Die Überlebensstrategien von Viren sind sehr unterschiedlich}

Die Replikationsstrategien von Viren sind außerordentlich unterschiedlich (akut, chronisch, persistierend). Wie bereits erwähnt (• Übersicht 2), müssen sich insbesondere pathogene Viren so verhalten, dass die Pathogenität nicht überhand nimmt und die Wirtspopulation Gefahr läuft, ausgerottet zu werden. Der evolutionäre Erfolg wird weiterhin determiniert von der Leichtigkeit der Übertragung (Kontagiosität) und von der Leichtigkeit der Ausbreitung, die der infizierte Wirt verursachen kann. Wirtsspezies mit hoher Populationsdichte und hoher Mobilität erleichtern Viren das Überleben.

Bei pathogenen Mikroorganismen kommt es letztlich für den evolutionären Erfolg auf eine optimale Korrelation zwischen Übertragungsfähigkeit und Virulenz an. Auch hier kann man 2 extreme Beispiele aus der Virologie zur Verdeutlichung anfügen:

- Das Tollwutvirus führt (unbehandelt) wahrscheinlich immer akut zum Tod des frisch infizierten Wirts. Also muss es stets schnell auf einen neuen Wirt übertragen werden. Hilfreich ist es deshalb für Tollwutviren, dass sie ein sehr breites Wirtsspektrum (Tropismus) haben, nämlich fast alle Warmblüter, den Menschen eingeschlossen, infizieren können. Nach Übertragung wandert das Virus sowohl in das zentrale Nervensystem (ZNS) als auch in die Speicheldrüsen, wo es sich vermehrt. Über die Infektion des ZNS kommt es zu einer Verhaltensänderung beim infizierten Tier (oder Menschen): Es entwickelt sich eine stark erhöhte Aggressivität und damit beim Tier, und übrigens auch beim infizierten Menschen, ein er- höhtes Beißverhalten. Da das Tollwutvirus sich gleichzeitig in den Speicheldrüsen vermehrt und im Speichel in hohen Titern vorhanden ist, sorgt dieses Virus so für eine möglichst zügige Übertragung, typischerweise innerhalb von 23 Wochen nach Infektion, auf einen neuen Wirt, bevor der vorherige Wirt an der Tollwut verstirbt.

- Ganz anders verhält sich z. B. das humane Immundefizienzvirus, dessen Kontagiosität zum Glück relativ gering ist. Dafür persistiert dieses Retrovirus lebenslang im Menschen und kann es sich leisten, sexuell nur relativ ineffizient übertragen zu werden. Genaue Untersuchungen bei HIV-diskordanten Paaren haben gezeigt, dass im Durchschnitt an die 500 ungeschützte Sexualkontakte notwendig waren, bis HIV 50\% der zuvor nicht infizierten Partner infiziert hatte (das heißt aber nicht, dass es nicht auch beim allerersten sexuellen Kontakt passieren könnte). Deshalb ist HIV auch keinesfalls daran interessiert, einen frisch infizierten Wirt zu schnell krank zu machen, da es sonst seine eigene Übertragungsmöglichkeit gefährden würde. Die in unseren Ländern zu beobachtende lange Latenzzeit zwischen primärer HIV-Infektion und dem Ausbruch von AIDS (unbehandelt durchschnittlich 10 Jahre) gibt HIV ausreichend Zeit, erneut übertragen zu werden.

\section{Übertragung zwischen den Spezies}

Die Medizingeschichte zeigt, dass die Transspezies-Transmission von Viren auch im 20. Jahrhundert, in dem solche Übertragungen natürlich besser denn je zuvor wissenschaftlich untersucht und dokumentiert werden konnten, zu zum Teil verheerenden Epidemien oder Pandemien geführt haben. Dazu einige Beispiele:

- Influenzaviren können die Speziesbarriere zum Menschen direkt überspringen. Ein Beispiel ist das Auftreten des A/H5N1-Virus 1997 in Hongkong und 2003 in Südchina sowie 2004 in einer Reihe von asiatischen Ländern oder, wie schon erwähnt, von $\mathrm{A} / \mathrm{H}_{7} \mathrm{~N} 7$ ebenfalls im Jahr 2003 in den Niederlanden. Eine Übertragung des Erregers von Mensch zu Mensch fand aber noch nicht 
oder nicht in nennenswertem Umfang statt; das Virus hatte sich offenbar noch nicht ausreichend an den neuen Wirt angepasst. Die Neuzusammensetzung (Reassortment) ganzer Gensegmente in tierischen „Mischgefäßen “ führte bisher mindestens 2-mal, 1957 und 1968, zu Pandemien (einer weltumspannenden Epidemie). 1968 z. B. trat ein ganz neues Virus auf, das sich wahrscheinlich aus einem menschlichen $\mathrm{A} / \mathrm{H}_{2} \mathrm{~N}_{2}$-Virus und einem Vogel-A/H3 $\mathrm{N}_{2}$-Virus zusammensetzte. Das resultierende $\mathrm{A} / \mathrm{H}_{3} \mathrm{~N}_{2}$-Virus löste eine Pandemie aus. Das Schwein gilt als besonders effektives „Mischgefäß" bei der Produktion neu kombinierter Viren, weil es Rezeptoren für menschliche und aviäre Influenzaviren hat. Aber auch Doppelinfektionen beim Menschen besitzen das theoretische Potenzial zum Reassortment.

- AIDS wird durch HIV verursacht, und HIV stammt von Altweltaffen ab (dort SIV genannt: simianes Immundefizienzvirus). Das weltweit am häufigsten vorkommende HIV-1 ist nach unserem heutigen gut fundierten Kenntnisstand von Schimpansen auf den Menschen übergegangen. Aber die natürlichen Wirte sind afrikanische Affen - die Rotkopf-Mangabe und die Große Weißnasenmeerkatze. Schimpansen jagen diese kleineren Affen und verzehren sie. Im Lauf der Zeit entstand in den Schimpansen eine Hybridform der beiden SI-Viren, die dann später auf den Menschen übertragen wurde. HIV-1 schaffte es höchstwahrscheinlich schon in den 3oer- bis 4oer-Jahren des letzten Jahrhunderts, auf den Menschen überzuspringen. Es verbreitete sich zunächst nur langsam in Zentralafrika. Die weltweite Verbreitung von HIV-1 begann etwa 1975. Der Ursprung von HIV-2, das vor allem in Westafrika verbreitet ist, ist das SIVsm der Rauchgrauen Mangabe (engl. Sooty mangabey). SIV verursacht in seinen natürlichen Wirten keine Erkrankung, d. h., über Jahrtausende oder noch länger haben sich SIV und sein Wirt miteinander dergestalt arrangiert, dass der natürliche Wirt nach der Infektion gesund bleibt und damit SIV auch über einen längeren Zeitraum auf andere Affen übertragen kann. Affenarten, die während der Evolution nicht mit

Tabelle 3

Beispiele viraler Mutationsraten

\begin{tabular}{ll} 
Virus & Mutationsrate pro Nukleotid pro Vermehrungszyklus \\
\hline Poliovirus & $10^{-6}$ \\
\hline Influenza-A-Virus & $10^{-5}$ \\
\hline Humanes Immundefizienzvirus & $10^{-4}$ \\
\hline
\end{tabular}

Tabelle 4

Neuartige virale Erkrankungen (Beispiele)

\begin{tabular}{ll}
\hline $\begin{array}{l}\text { Infektion bzw. Erreger } \\
\text { Humanes Immundefizienzvirus }\end{array}$ & $\begin{array}{l}\text { Faktoren, die die Übertragung und/oder Krankheits- } \\
\text { entstehung begünstigen }\end{array}$ \\
\hline (HIV)/AIDS & $\begin{array}{l}\text { Migration in Städte, Reisetätigkeit, Promiskuität, Prostituti- } \\
\text { on, kontaminierte Blutprodukte und Spritzen }\end{array}$ \\
\hline Influenza & $\begin{array}{l}\text { Möglicherweise die gemeinsame Aufzucht von Geflügel } \\
\text { und Schweinen, was das Reassortment der Viren erleichtert }\end{array}$ \\
\hline Lassa u. a.hämorrhagische Fieber & $\begin{array}{l}\text { Urbanisierung mit Zunahme der virustragenden Ratten, } \\
\text { damit zunehmende Expositionsrate }\end{array}$ \\
\hline Gelbfieber, Dengue-Fieber & Zunahme des Zwischenwirts Moskito \\
\hline $\begin{array}{l}\text { Bovine Spongiforme Enzephalopathie } \\
\text { (BSE) }\end{array}$ & Veränderungen in der Futtermittelherstellung \\
\hline
\end{tabular}

SIV in Berührung gekommen sind, wie Rhesusaffen, erleiden bei (z. B. experimentellen) SIV-Infektionen das gleiche Schicksal wie der HIV-infizierte Mensch - als nicht-natürliche Wirte entwickeln sie simianes AIDS (SAIDS). Es ist nicht bekannt und daher eines der zentralen Themen der AIDS-Forschung, warum natürliche Wirte lebenslang SIV tragen und dennoch gesund bleiben, heterologe Wirte (z. B. Mensch, Rhesusaffe) unbehandelt jedoch AIDS entwickeln und versterben. Gegenwärtig zählt die AIDS-Forschung mehr als 2 Dutzend Affenarten in Afrika, die natürlicherweise SIV infiziert sind. Nur 2-mal haben sich SIV-Stämme bisher als HIV-1 und HIV2 im Menschen etablieren können; wahrscheinlich erfolgte die TransspeziesÜbertragung durch gejagte Affen und über Hautverletzungen des Jägers. Wenig ermutigend ist die Vorstellung, weitere andere SIV-Stämme könnten ebenfalls noch auf den Menschen übertragen werden, mit unbekannten epidemiologischen und medizinischen Konsequenzen. Jetzt schon wird das Auftreten von AIDS als größte medizinische Katastrophe der Neuzeit gewertet.

- Auch der Rinderwahnsinn, BSE, ist möglicherweise eine Transspezies-
Transmission. Ein häufig zitierter Erklärungsansatz ist, dass Scrapieerreger infolge geänderter Produktionsbedingungen bei der Futtermittelherstellung akzidentell vom Schaf auf das Rind übertragen wurden und sich dann an die neue Wirtsspezies adaptiert haben. Nach der Prionhypothese wäre aber auch vorstellbar, dass BSE im Rind durch eine spontane oder genetisch bedingte Fehlfaltung des Prionproteins und den Übergang in eine infektiöse Isoform de novo entstanden ist. Dennoch steht außer Frage, dass, wie schon beschrieben, die Verfütterung nicht ausreichend inaktivierter Tiermehle und möglicherweise auch Tierfette für die Verbreitung entscheidend waren. Diese Transmissible Spongiforme Enzephalopathie (TSE) zeigt ein beunruhigend breites Wirtsspektrum. Futtermittel aus BSE-infizierten Rindern führten zu Infektionen von Katzen, Löwen und Panthern mit dem BSE-Erreger und zur Entwicklung einer TSE-Erkrankung. Bei dieser ungewöhnlich breiten oralen Übertragbarkeit des TSE-Erregers ist es nicht verwunderlich, dass sich auch der Mensch in bisher zahlenmäßig zum Glück seltenen Fällen mit dem BSE-Erreger infizierte. 
Übersicht 5

Beispiele des Selektionsdrucks auf Viren

Wirtszellbereich (Tropismus) wird reguliert durch

- Virale und zelluläre Rezeptoren

- Genetische Resistenz des Wirts

- Transkriptionelle Regulation der viralen Replikation durch die Wirtszelle

- Wirtszellenzyme (Methylasen, Proteasen, Glycosidasen, Glycosyltransferasen)

- Die Immunabwehr des Wirts

Regulation der Virusreplikation durch

- Virale Polymerasen

- Virale genomische Erkennungssequenzen für virale und zelluläre regulatorische Proteine

- Virale RNA Spleiss-Sequenzen (alternatives Spleissen)

Virale Virulenz

- Oft abhängig von mehreren viralen Funktionen

- Oft abhängig von Enzymen der Wirtszelle

Übersicht 6

Infektionskrankheiten - Präventionsstrategien

Verbesserte öffentliche Gesundheitsfürsorge (insbesondere im Trinkwasser-/Abwasserbereich)

Technologiefolgenabschätzung

Weitere und verbesserte Impfstoffe

Weitere und verbesserte, insbesondere antivirale Therapien

Verbesserte Kontrolle und ggf. Elimination der Zwischenwirte (Mücken, Nager)

Globales Überwachungssystem (Surveillance)

Im Übrigen ist es nicht selten, dass die natürlichen Wirte eines Erregers sich mit dem Erreger evolutionär dergestalt arrangiert haben, dass die Erreger sich vermehren dürfen, der Wirt aber nicht krank wird. Mit einem Spezieswechsel kann aber auch (s. oben) eine erhöhte Pathogenität einhergehen.

\section{Neuartige Viren und Selektionsdruck}

Wie bereits erwähnt, besitzen insbesondere RNA-haltige Viren eine sehr hohe Mutationsrate (- Tabelle 3). Es stellt sich deshalb die Frage, warum bei der bekannt hohen Mutationsrate nicht noch viel häufiger neuartige Viren entstehen und sich ausbreiten.

Es gibt einen großen Selektionsdruck, der das Überleben und erst recht die Ausbreitung mutanter Viren beschränkt. Viren sind in ihrer parasitären Beziehung zur Wirtszelle außerordentlich spezialisiert. Fast jeder Schritt im Lebenszyklus des Virus ist abhängig von komplementären Eigenschaften der
Wirtszelle, die diesen Schritt ermöglichen muss.

So sind z. B. Mutationen im 3-dimensionalen Virusrezeptor, der zum Andocken an die Wirtszelle dient, meistens tödlich für das Virus. Nach Einschleusen des Viruserbmaterials in die Zelle ist dessen Vermehrung abhängig von positiv regulierenden zellulären Transkriptionsfaktoren, die, um die virale Replikation zu ermöglichen, unveränderte Erkennungssequenzen auf dem viralen Genom benötigen. Virale Enhancer- und Promoterregionen dürfen ebenso wenig stark mutiert sein wie virale RNA-Spleiss-Sequenzen. Die viralen Proteine werden häufig noch von zellulären Enzymen (Methylasen, Proteasen, Glycosyltransferasen und Glycosidasen) modifiziert, was normalerweise nur möglich ist, wenn die entsprechenden viralen Gene nicht extensiv mutiert sind. Unabhängig von der viralen Mutationsfähigkeit wird Virusvirulenz häufig von mehreren Genen bestimmt. Mutation in einem Gen kann zum vollständigen Verlust der Virusvirulenz führen. Oft ist auch die Ausprägung der Vi- rusvirulenz noch abhängig von posttranslationalen Modifikationen durch zelluläre Enzyme.

Da einerseits viele Virusfamilien evolutionär sehr erfolgreich, andererseits aber genomisch klein und in ihrem Vermehrungszyklus kompliziert sind, wird deutlich, dass fast alle Mutationen von evolutionärem Nachteil sind. Beispiele für den Selektionsdruck auf die Virusevolution sind in der $\bullet$ Übersicht 5 zusammengefasst.

\section{Prävention und Bekämpfung neuer Infektionskrankheiten}

Neuartigen Infektionskrankheiten, z. B. den in den letzten Jahren so „erfolgreichen" (aus der Sicht der Viren) Virusinfektionen (๑ Tabelle 4 ), sind wir selbstverständlich nicht schutzlos ausgeliefert.

Zum einen kann und muss man die beeinflussbaren Ursachen, die die Übertragung oder Krankheitsentstehung begünstigen, bekämpfen (• Tabelle 4).Zum anderen gibt es in den Ländern mit einem hohen Medizinstandard ein breites Arsenal an Möglichkeiten zur Infektionsbekämpfung. Wie bereits eingangs erwähnt, verfügen wir über Impfstoffe und Antibiotika gegen bakterielle Infektionen sowie über Medikamente gegen parasitäre und viral Erkrankungen. Vor allem gibt es in industrialisierten Staaten im Allgemeinen eine hohe individuelle Hygiene und sehr hohe öffentliche und medizinische Hygienestandards.

In den Ländern jedoch, in denen der medizinische Standard nicht so hoch ist, sind sowohl die diagnostischen und therapeutischen Möglichkeiten der Bekämpfung von Infektionskrankheiten eingeschränkt als auch die medizinischen Maßnahmen durch Nicht-Einhalten von Hygieneregeln selbst eine potenzielle Infektionsquelle. Die Wiederverwendung von nicht ausreichend sterilisierten medizinischen Instrumenten oder Injektionsnadeln hat z. B. in Ägypten in der 2. Hälfte des vergangenen Jahrhunderts zur größten iatrogenen HCV-Epidemie geführt, und auch bei der Ausbreitung von HIV in Entwicklungsländern spielen diese Übertragungswege sowie Transfusionen von nicht ausgetestetem Blut ebenfalls eine große Rolle. Deshalb, und weil die Me- 
gastädte der Dritten Welt von den Experten als die potenziellen Ausgangsorte für neue Epidemien angesehen werden, muss es in erster Linie dort zu einer Verbesserung der öffentlichen Gesundheitsvorsorge kommen (• Übersicht 6). Man konnte es bereits vor über 100 Jahren in Europa erleben, in neuerer Zeit auch in den sich entwickelnden Ländern: Allein die Einführung einer einwandfreien Trinkwasserversorgung und die Klärung der Abwässer führt zu einer drastischen Reduktion oral übertragener Infektionen.

Was wir weiterhin brauchen, ist eine verbesserte Technologiefolgenabschätzung, um die Risiken, die sich aus dem menschlichen Handeln ergeben, möglichst vorab kalkulieren zu können. Darüber hinaus sollten Impfstoffe und Therapeutika zur Behandlung viraler (und parasitärer) Infektionen weiterentwickelt werden. Diese müssen auch in den Entwicklungsländern zur Verfügung stehen, ebenso wie das Know-how und die Technologie, um Transfusionen infektionssicher zu machen, insbesondere in Gebieten mit hoher Hepatitis- und HIVPrävalenz.

Die epidemiologische Überwachung, die in den letzten Jahren von der WHO in Genf im Sinne eines Netzwerks etabliert worden ist, trägt zur Früherkennung von Infektionen bei und hilft entscheidend, rechtzeitig notwendige Maßnahmen zur Vermeidung einer Ausbreitung zu ergreifen. In diesem Netzwerk kooperieren unter anderen Mikrobiologen und Kliniker, um vor allem die Slums der tropischen Megastädte zu überwachen. Auf Anforderung können internationale mobile Arbeitsgruppen aus Klinikern und fachkundigen Wissenschaftlern Verdachtsfällen nachgehen. Das Informationsnetz wird ausgebaut, um ähnlich wie bei der Früherkennung neuer Influenzavirusstämme möglichst rechtzeitig neue bzw. neuartige Infektionen diagnostizieren zu können.

Betrachten wir die Evolution, so sind es eigentlich nur die Mikroorganismen und unter diesen insbesondere die Viren, die evolutionär mindestens ebenso erfolgreich waren wie der Mensch. Wir werden uns auch zukünftig auf die Veränderungsfähigkeit von Mikroorganismen, insbesondere von Viren, verlassen können. Deshalb liegt es am Handeln des
Menschen sicherzustellen, dass Viren evolutionär nicht noch erfolgreicher werden als bisher und damit das Bedrohungspotenzial für den Menschen erhöhen können. Eine AIDS-Epidemie sollte uns Lehre genug sein.

Dieser Beitrag beruht in Teilen auf dem Vortrag „Die Renaissance alter und neuer Seuchen als Konsequenz menschlichen Handelns" im Rahmen der Akademievorlesung der Berlin-Brandenburgischen Akademie der Wissenschaften.

\section{Korrespondierender Autor \\ Prof. Dr. R. Kurth}

Robert Koch-Institut, Nordufer 20, 13353 Berlin E-Mail:kurthr@rki.de

\section{Danksagung}

Ich danke Frau Susanne Glasmacher und Frau Dr. Ruth Offergeld (beide Robert Koch-Institut) für ihre redaktionelle Mitarbeit bei der Erstellung dieses Manuskripts.

\section{Weiterführende Literatur}

Beeson PB (1943) Jaundice occurring one to four months after transfusion of blood or plasma. JAMA 121:13321334

Biggerstaff BJ, Petersen LR (2002) Estimated risk of West Nile virus transmission through blood transfusion during an epidemic in Queens, New York City. Transfusion 42:1019-1026

Centers of Disease Control and Prevention (1994) Addressing emerging infectious disease threats: a prevention strategy for the United States. Morb Mort Wkly Rep 43 (RR-5):1-18

Cohen ML (1992) Epidemiology of drug resistance: implications for a post-antimicrobial era. Science 257:10501055

Denner J (2001) Xenotransplanation, Transspezies-Übertragung von Retroviren und AIDS. In: Arndt D, Obe G, Kleeberg $\mathrm{U}$ (Hrsg) Biotechnologische Verfahren und Möglichkeiten in der Medizin. (RKI-Schrift 1/01) Urban \& Vo gel Medien und Medizin Verlagsgesellschaft, München, S 164-183

Denner J, Kurth R (2001) Infektionen bei Immunschwächen (einschließlich AIDS). In: Köhler W, Eggers HJ, Fleischer B et al. (Hrsg) Medizinische Mikrobiologie. Verlag Urban \& Fischer, München, S 793-811

Frank C, Mohamed NK, Strickland GT et al. (2000) The role of parenteral antischistosomal therapy in the spread of hepatitis C virus in Egypt. Lancet 11;355(9207):887-891

Holland JJ (1996) Evolving virus plagues. Proc Natl Acad Sci USA 93:545-546

Hunter N, Foster J, Chong A et al. (2002) Transmission of prion disease by blood transfusion. J Gen Virol 83:28972905

Krause RM (1981) The restless tide: the persistent challenge of the microbial world. The National Foundation for Infectious Diseases, Washington, D.C.

Kunin CM (1993) Resistance to antimicrobial drugs - a worldwide calamity. Ann Internal Med 118:557-560
Kurth R (1991) Infektionskrankheiten der Zukunft. AIDS-Forschung 6:178-185

Kurth R (2000) Die Renaissance alter und neuer Seuchen als Konsequenz menschlichen Handelns. In: Berlin-Brandenburgische Akademie der Wissenschaften (Hrsg) Berichte und Abhandlungen, Band 8. Akademie Verlag, Berlin

Kurth R (1992) Infektionskrankheiten im Wandel. Diagnose Labor 42:47-58

Lederberg J, Shape RE, Oaks Jr SC (1992) Emerging infections: microbial threats to health in the United States. National Academy Press, Washington, D.C

Lederberg J (1997) Infectious disease as an evolutionary paradigm. Emerg Infect Dis 3:417-423

Levy SB (1993) Confronting multidrug resistance: a role for each of us. JAMA 269:1840-1842

Morse SS (1993) Emerging viruses. Oxford University Press, New York 76:599-601

Murphy FA (1994) New, emerging, and reemerging infectious diseases. Adv Virus Res 43:1-52

Ragni MV, Lewis JH, Spero JA, Bontempo FA (1983) Aquiredimmunodeficiency-like syndrome in two haemophiliacs. Lancet 1(8318):213-214

Witte W (1998) Medical consequences of antibiotic use in agriculture. Science 279:996-997 